\title{
Surveillance for acute cellular rejection after lung transplantation
}

\author{
Mark Greer ${ }^{1,2}$, Christopher Werlein ${ }^{3}$, Danny Jonigk ${ }^{2,3}$ \\ ${ }^{1}$ Department of Respiratory Medicine, Hannover Medical School, Hannover, Germany; ${ }^{2}$ Biomedical Research in End-Stage and Obstructive Lung \\ Disease (BREATH), German Centre for Lung Research (DZL), Hannover, Germany; ${ }^{3}$ Institute for Pathology, Hannover Medical School, Hannover, \\ Germany \\ Contributions: (I) Conception and design: M Greer; (II) Administrative support: M Greer, C Werlein; (III) Provision of study materials or patients: \\ C Werlein, D Jonigk; (IV) Collection and assembly of data: M Greer, C Werlein; (V) Data analysis and interpretation: All authors; (VI) Manuscript \\ writing: All authors; (VII) Final approval of manuscript: All authors. \\ Correspondence to: Mark Greer. Department of Respiratory Medicine, Hannover Medical School, Hannover, Germany; Biomedical Research in End- \\ Stage and Obstructive Lung Disease (BREATH), German Centre for Lung Research (DZL), Hannover, Germany. Email: greer.mark@mh-hannover.de.
}

\begin{abstract}
Acute cellular rejection (ACR) is a common complication following lung transplantation (LTx), affecting almost a third of recipients in the first year. Established, comprehensive diagnostic criteria exist but they necessitate allograft biopsies which in turn increases clinical risk and can pose certain logistical and economic problems in service delivery. Undermining these challenges further, are known problems with inter-observer interpretation of biopsies and uncertainty as to the long-term implications of milder or indeed asymptomatic episodes. Increased risk of chronic lung allograft dysfunction (CLAD) has long been considered the most significant consequence of ACR. Consensus is lacking as to whether this applies to mild ACR, with contradictory evidence available. Given these issues, research into alternative, minimal or noninvasive biomarkers represents the main focus of research in ACR. A number of potential markers have been proposed, but none to date have demonstrated adequate sensitivity and specificity to allow translation from bench to bedside.
\end{abstract}

Keywords: Lung transplant; rejection; bronchoscopy; histopathology

Submitted Nov 25, 2019. Accepted for publication Feb 19, 2020.

doi: 10.21037/atm.2020.02.127

View this article at: http://dx.doi.org/10.21037/atm.2020.02.127

\section{Introduction}

Acute cellular rejection (ACR), as its name implies is a histological diagnosis referring to de novo perivascular and interstitial infiltration of mononuclear cells within the allograft (1). Traditional thinking has considered ACR to predominantly occur in the early post-transplantation period, with International Society for Heart and Lung Transplantation (ISHLT) registry data suggesting that $27 \%$ of patients experience $\geq 1$ episodes in the first year (2). Interestingly, there has been a small but steady decline in ACR reported rates in recent years. Due to methodological as well as reporting issues, these figures may well underestimate incidence, as highlighted in a couple of prospective trials comparing different maintenance immunosuppression regimes, where rates of ACR in excess of $45 \%$ were reported $(3,4)$.
In this review, the underlying immunological mechanisms will be summarized as a basis for discussing known risk factors implicated in causing ACR. The clinical presentation and its incumbent ambiguities will be outlined and the wide-ranging issues surrounding investigation and diagnosis will be considered. Thereafter the role of surveillance screening, including potential novel biomarkers will be evaluated, before finally treatment both in terms of indications and goals will be reviewed.

\section{Host responses to the allograft}

The alloimmune host versus graft response is predominantly driven by T-lymphocytes and their recognition of circulating or tissue resident major histocompatibility complex (MHC) antigens. The latter bind and present 


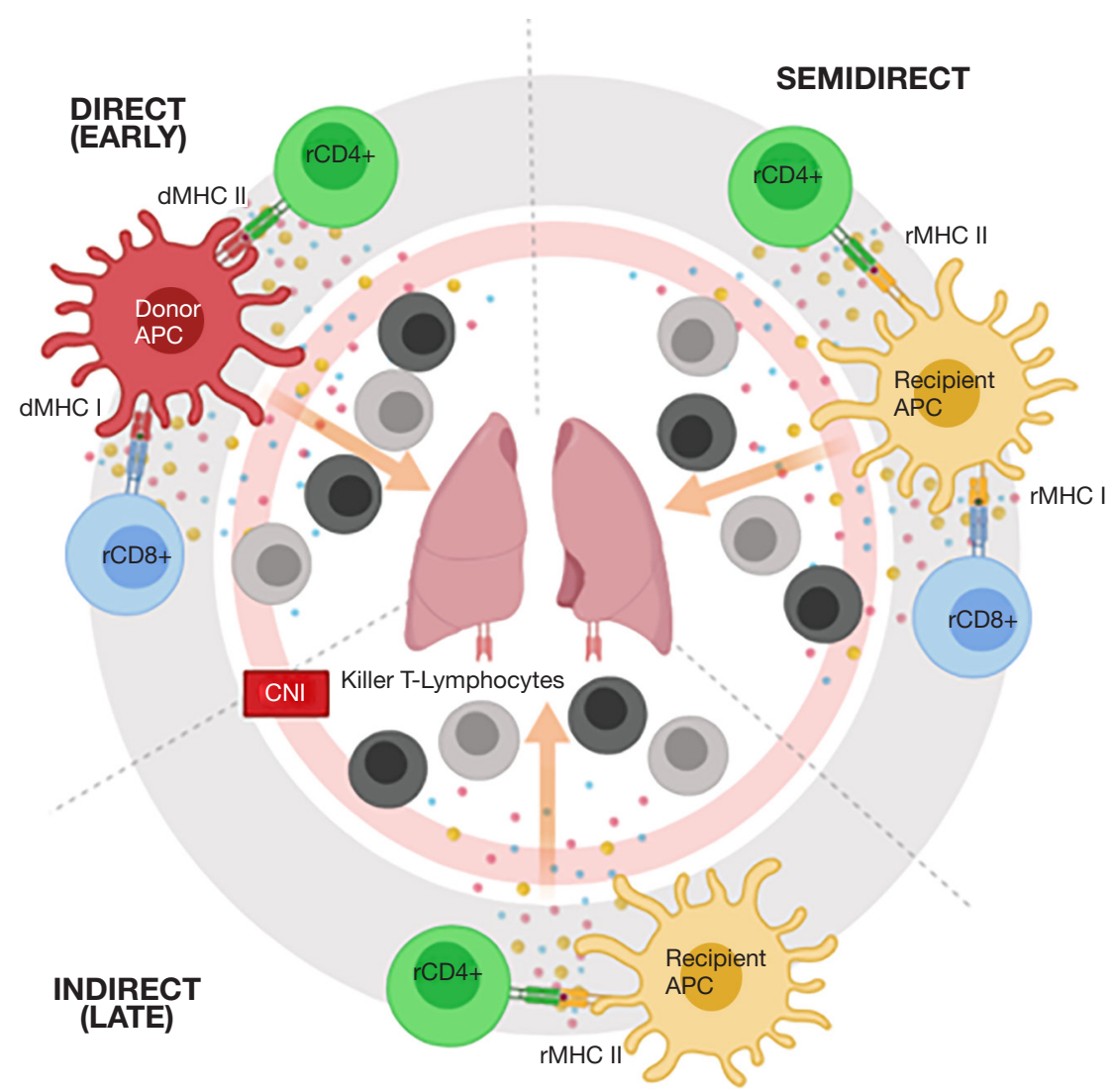

Figure 1 Summarizing the pathways of recognition for donor alloantigens by recipient T-lymphocytes. Three co-existing pathways are known. Initially recipient T-cells are activated by intact donor MHC molecules on passenger donor antigen-presenting cells via a direct pathway. Over time this is succeeded by host antigen-presenting cells (APC) responses to processing of immunogenic apoptotic donor cell remnants activating MHC class II-restricted recipient CD4 lymphocytes via the indirect pathway. The semi-direct pathway represents a variant of the latter in which recipient APCs have been shown to exchange cell membrane components, including MHC molecules that can facilitate additional CD8 lymphocyte activation. The maturation and differentiation of such lymphocytes is known to be inhibited by calcineurin inhibitors (CNI). dMHC, donor major histocompatibility complexes; rMHC, recipient major donor histocompatibility complexes; rCD4, recipient CD4 lymphocyte; rCD8, recipient CD8 lymphocyte.

foreign antigenic peptides on the surfaces of antigen presenting cells (APCs), facilitating their recognition by the former. Genes coding for these MHCs, often referred to as human leukocyte antigens (HLAs) in allografts, display enormous polymorphism leading to great individual diversity in humans. In allogenic transplantation, the degree of variance-or conversely matching-between donor and recipient HLAs appears critical in graft tolerance as this influences the degree of recognition between native and foreign tissue.

This allo-recognition is considered central to cellular rejection. It is however not considered a uniform process, and may implicate different immunological pathways to varying degrees at a given time point (Figure 1). In the initial phase post-transplantation, allograft tissue-resident donor dendritic cells (DCs) migrate towards secondary lymphoid tissues, presenting donor HLA directly to recipient T-lymphocytes via the direct pathway (5). Over time recipient DCs succeed dwindling donor DCs, expressing allo-antigens obtained from remaining donor APCs to the recipient T-lymphocytes. The latter, once activated, exhibit clonal expansion and differentiation into cytotoxic T-lymphocytes before migrating back into the allograft and binding with donor HLA triggering localized tissue injury (6). Recently, a variant of this indirect pathway allowing activation by MHC class I and translocated class II molecules on recipient antigen- 
presenting cells has been demonstrated and is referred to as the semi-direct pathway (7).

\section{Epidemiology \& risk factors}

ISHLT registry data has consistently reported slightly higher rates among younger adult recipients, independent of gender. Furthermore no differences in incidence have been observed between primary lung transplantation (LTx) and first redo LTx (2). The actual rates of ACR seen are somewhat higher compared to kidney (8) transplantation but lower than in liver transplantation (9) over the first year. Data obtained from multi-organ donors, has previously shown no association in ACR incidence between different organs from the same donor (10).

\section{Age \& its confounders}

In considering why age might influence ACR, several important contributing factors need to be taken into account. Younger LTx recipients are usually transplanted for very different indications than in older patients, which may influence the extent of residual extra-pulmonary disease. Furthermore, confounders such as lifestyle, social circumstances and pressures exist that may be difficult to quantify. Perhaps the simplest of these to assess is the influence of transplant indication and data exists indicating that adult patients with cystic fibrosis (CF) appeared to experience more frequent and more severe ACR than other disease groups (11). Age-dependent sub-analysis in both CF and non-CF populations showed decreasing incidence and ACR severity with increasing age in CF, but not in non-CF populations.

\section{Induction and maintenance immunosuppression}

Perioperative antibody induction therapy, particularly interleukin-2 receptor antagonists continues to gain popularity, with latest registry data reporting their use in $80 \%$ of adult LTx procedures (2). In terms of the effect of induction in reducing the risk of developing ACR, an early meta-analysis of 3 relatively small studies failed to demonstrate any superiority versus standard of care for antibody induction (12). More recent data however has suggested lower rates of ACR among recipients receiving the anti-CD52 receptor monoclonal antibody Alemtuzumab, compared to more traditional agents such as anti-thymocyte globulin (OR 0.21, 0.11-0.40; $\mathrm{P}<0.001)$ and basiliximab (OR $0.12,0.03-0.55 ; \mathrm{P}<0.01$ ), as well as fewer infections (13).

Maintenance immunosuppression usually consists of a triple therapy, combining a calcineurin inhibitor (CNI), a purine synthesis inhibitor and steroids. ISHLT registry data (2) shows that Tacrolimus has become the overwhelming CNI choice (>90\%), replacing cyclosporine. In terms of ACR, a single center randomized trial revealed little difference in the incidence of mild rejection between CNIs, but lower incidence of higher grade ACR in the first 3 years (36\% vs. $61 \%$ ) among patients receiving Tacrolimus (4).

\section{Treatment adberence}

Drug metabolism and treatment adherence are often cited as contributing to allograft injury and a comprehensive meta-analysis has been published previously (14). Few studies have compared actual drug adherence and clinical outcomes, with none addressing a relationship to ACR directly. Addressing adherence in broader terms, incorporating home spirometry and tobacco avoidance, the only demonstrated effect has been shortened CLAD free survival (15). Recent data focusing on interventions designed to improve adherence, did demonstrate an association between ACR and subsequent non-adherence (16). Whilst this conclusion may be limited by study design with regards to behavior prior to ACR, it reiterates the influence of aspects of patient behavior on outcomes.

\section{Role of gene polymorphism in the alloimmune response}

Given the central role of HLA encoding in alloimmune tolerance, differences between donor and recipient HLA have long been implicated in allograft rejection. As indicated above, these genes are subject to extensive polymorphism, leading to wide diversity in humans. It has been shown that such mismatches, in particular in HLA-A and potentially HLA-B may contribute to ACR, whereas HLA-DR as well as total number of mismatches did not influence outcomes (17). Non-HLA antibodies such as angiotensin Type 1 receptor antibodies have also been implicated in kidney and heart allograft outcomes, with pre-transplant titers showing trends towards increased risk of ACR, albeit without achieving significance (18).

Genetic polymorphism in alloimmunity is however not limited to HLA and has been shown to influence the expression and function of a wide variety of implicated immune-regulatory factors. A further example involves 
specific chemokines, which have been implicated as important mediators in graft tolerance. Genetic variation in molecules such as CCL4L have been confirmed in ACR, with correlation between increasing copy number variation and both likelihood and frequency of ACR (19).

Similar phenomena have been observed with certain cytokines. It has been shown that cytokines may assist in leucocyte recruitment and maturation in alloimmunity, demonstrating both favorable and unfavorable influences on allograft tolerance. Specific toll-like receptors (TLRs), involved in innate immune responses to microbial pathogens are known to be abundantly expressed in airway epithelia (20) and genetic polymorphisms in the recipient TLR-4 gene, rather than that of the donor have been found to be protective in terms of ACR (21-23).

Likewise, single nucleotide polymorphisms (SNP) within the complement system have demonstrable influence on alloimmunity. Evidence exists for C3 complement which is involved in all 3 immune pathways. Although predominantly produced in the liver, C3 may also originate from alveolar macrophages and type II pneumocytes. Two C3 molecular variants exist, differentiated according to their responses to electrophoresis: fast $v s$. slow, with the latter being more prevalent in humans (20:80). LTx recipients that are homozygotes for slow $\mathrm{C} 3$ (SS), but not donors have exhibited higher rates of ACR (HR 6.2, 1.4-27.1; $\mathrm{P}=0.015$ ) (24).

The influences of SNPs in Fc gamma receptors in ACR have similarly been reported. These participate primarily in humoral responses, and in particular antibody-dependent cytotoxic alloimmune responses of natural killer (NK) cells. It has been however previously reported that the FCGR3A $[158 \mathrm{~V} / \mathrm{V}]$ genotype was associated with early ACR (HR 4.8, 2.4-9.6; $\mathrm{P}<0.001)$, but not $\mathrm{NK}$ cell activation in response donor-specific HLA, whereas FCGR2A [131R/R] was associated with earlier CLAD and early development of donor-specific antibodies (25).

The impact of such genetic variability is however not limited to direct influences of alloimmune responses. SNPs have also been implicated in genes responsible for drug metabolism. A relevant example in LTx, relates to published data for mycophenolic acid (MMF), in which genotyping of targeted SNPs known to regulate MMF were compared to clinical outcomes. It could be shown that specific polymorphisms contributed to higher incidence of ACR, as well as increased 1- and 3-year graft loss in patients receiving MMF but not those with the same genotypes receiving azathioprine (26).

\section{Infection}

\section{Cytomegalovirus (CMV)}

CMV has long been associated with allograft injury in solid organ transplantation, with activation of Th1 alloimmune responses having been implicated $(27,28)$. The constellation of donor and recipient CMV serostatus is known to influence this risk, with seropositive allografts transplanted into seronegative recipients carrying the greatest risk (29). Reported rates of CMV disease of between $50-91 \%$ (30) have led many centers to initiate $\mathrm{CMV}$ prophylaxis strategies of varying forms and duration. The emergence in recent years of ganciclovir resistant CMV infections has to some extent reignited old concerns. Preliminary data with regard to ACR in such patients has to date however shown no meaningful differences in terms of outcomes (31).

\section{Community-acquired respiratory viruses (CARV)}

Although often suspected, clear evidence demonstrating a causal link in infect-triggered acute rejection is inconclusive. While numerous reports addressing this issue, have discounted such associations (32-35), a large single center study, that involved more than 1,000 nasopharyngeal swabs analyzed by PCR over 5 years as part of a routine surveillance program. This concluded that in the 3 months following a CARV, that a 6-fold increase in the risk of ACR was present (HR 6.5, 1.5-29.1; $\mathrm{P}=0.01$ ). Sub-analysis suggested links to both direct responses to viral replication as well as the deleterious sequelae due to the release of Th1- and Th2-type alloreactive cytokines in response to graft injury (36).

Others have implicated CARV infections in triggering the release of allograft resident exosomes as a consequence of graft injury (37). Such exosomes have also been observed in ACR and are known to express HLA antigens and lung-associated self-antigens (SAgs). This introduces the possibility of synergistic roles for both alloimmunity and autoimmunity within the context of graft injury (38). Induction and circulatory release of cell membrane vesicles, known as exosomes containing specific lung SAgs for collagen-V $(\mathrm{Col}-\mathrm{V})$ and $\mathrm{K}$-alpha 1 tubulin $(\mathrm{K} \alpha 1 \mathrm{~T})$ have demonstrated 9.5-fold and 12.6-fold increases in risk of ACR respectively (37). 


\section{Leucopenia and granulocyte colony stimulation factors $(G-C S F)$}

Neutropenia occurs frequently after LTx, most commonly in the early post-operative phase. In a recent single center analysis, neutrophil counts below $1.5 \mathrm{Ts} d / \mu \mathrm{L}$ were observed in more than $40 \%$ of LTx recipients. Severe neutropenia $(<500 / \mu \mathrm{L})$ had higher mortality rates but no association to ACR (39). Similarly another study assessing the use of G-CSF, revealed a slightly higher incidence of ACR among those requiring treatment but this failed to achieve significance ( $57 \%$ vs. $51 \% ; \mathrm{P}=0.48)$. Likewise no differences in ACR severity were seen (40).

\section{Clinical sequelae}

Clinically apparent ACR generally involves acute or subacute dyspnea developing over several hours or days. It can be accompanied by hypoxia, cough and occasionally fever. The severity of symptoms varies widely, from completely asymptomatic patients to constellations akin to adult respiratory distress syndromes including "white out" (41). Structured data correlating degree of symptoms with ACR grade has been previously published (42). In centers performing routine home spirometry, asymptomatic losses in graft function are not uncommon and are considered clear indications warranting further urgent investigation. In all its guises however, there is no pathognomonic presentation that distinguishes ACR from other common early complications such as viral or even bacterial infections.

\section{Peripheral blood}

Routine laboratory analysis is recommended, but its main role is to corroboratively discount other possible causes of acute graft dysfunction. Whereas new-onset peripheral neutrophilia has been suggested to favor an infectious cause, varying increases in different leukocyte populations including lymphocytes, eosinophils and basophils have previously been reported in ACR, but all have lacked any sense of useful specificity to aid with diagnosis (43-46).

\section{Spirometry}

In symptomatic patients a measurable decline in graft function is usually apparent. In terms of typical spirometric patterns, similar reductions in forced expiratory volume in $1 \mathrm{~s}\left(\mathrm{FEV}_{1}\right)$, forced vital capacity (FVC) and total lung capacity (TLC) have been found (47). These findings proved non-discriminatory with regards to common alternative causes such as acute infection. Arbitrarily, a cutoff of $\geq 10 \%$ loss in $\mathrm{FEV}_{1}$ persisting $\geq 48 \mathrm{~h}$ has become the established mandate for further investigation (48). Obvious advantages exist in centers advocating home spirometry, allowing early detection and even quantification of clinically asymptomatic loss in graft function. Some centers have even trialed telemedicine, with devices autonomously triggering warnings at the treatment center (49). Concerns have been raised about reliability among unilateral-LTx recipients, where disease progress in the remaining native lung can confound interpretation (50). Moreover, it is important to reiterate how commonly ACR occurs in the absence of acute graft dysfunction, with rates approaching $40 \%$ of all cases having been reported (51).

\section{Imaging}

A variety of imaging techniques have been investigated as potential discriminators of ACR, but to date none have demonstrated consistent reliability in terms of accuracy or indeed severity, to have shifted standard practice. Previously published data assessing chest X-ray (CXR) and computed tomography (CT) has suggested specificity of around $70 \%$, with sensitivity being as low as $35 \%$ (52), suggesting that the utility of imaging remains primarily the exclusion of other causes such as pneumonia or tumors. More recently, tailored high-resolution CT protocols were compared to trans-bronchial lung biopsies in 26 patients (53). Certain features on CT, such as ground-glass opacities, inter-lobar septal thickening, particularly when involvement is bilateral and predominantly basal, have a reported accuracy of almost $90 \%$ within the small sample cohort. A recent, somewhat larger retrospective analysis of 78 patients has again cast some doubt on the utility, but failure to distinguish between symptomatic and asymptomatic patients in the latter may have influenced their findings (54). Undoubtedly, sensitivity remains an important weakness and in themselves the features mentioned, as well as pleural or pericardial effusions in the absence of further evidence of cardiac decompensation, should increase suspicion, but are in themselves not diagnostic and demand further investigation (55).

Beyond standard CXR and HRCT, research into other non-invasive, hybrid imaging modalities has suggested some merit in both magnetic resonance imaging (MRI), single photon emission computed tomography (SPECT) and positron-emission tomography (PET). Conceptually, 
techniques assessing dynamic changes in both anatomy and tissue metabolism are clearly attractive in the setting of ACR. Data in this setting is currently limited to preclinical animal models, however promising results with hyperpolarized pyruvate intravenous contrast or ultra-short echo MRI in rat and mouse models have been reported $(56,57)$. In a recent clinical proof-of-concept study using ${ }^{99}$ Tc-HYNC-IL-2 SPECT scanning, radio-labelled Interleukin-2 (IL-2) targeting high-affinity IL-2 receptors on activated T-lymphocyte populations involved in ACR was assessed (58). In total 3/13 patients fulfilled current criteria for ACR, of which two were correctly identified. While one patient with milder ACR was missed, no false positives were noted. Earlier PET data, attempted to distinguish ACR from infectious causes by measuring ${ }^{18} \mathrm{~F}$-fluorodexoyglucose $\left({ }^{18} \mathrm{FDG}\right)$ uptake to quantify neutrophil activity that is characteristically absent in ACR (59). Eight patients had biopsy-proven ACR, of whom five had signs and symptoms suggestive of mixed etiology. Among those with isolated ACR, ${ }^{18}$ FDG uptake was identical to healthy controls.

Although continued research into diagnostic imaging is essential. It raises a number of other relevant issues, ranging from local resources in terms of equipment, system capacity, logistics and expertise in such a niche area, to harming patients in less apparent ways through additional exposure to various contrast mediums and indeed ionizing radiation than current surveillance.

\section{Bronchoscopy}

The role of bronchoscopy in LTx is uniquely multi-faceted, extending beyond a mere sampling device. Particularly in the early post-transplant phase, inspection of the anastomoses and airway healing may be considered prudent, with debridement proving to be necessary upon occasion (60-62). With particular regard to ACR, a number of factors require careful consideration.

Rarely is the clinical suspicion of ACR completely unequivocal, and in practice simultaneous evaluation of the various common etiologies is required. Bronchoscopy facilitates rapid discrimination between likely causes and helps prevent the potentially harmful consequences of an erroneous treatment decisions, with particular regard to adjusting immunosuppression.

Clearly in such symptomatic patients, bronchoscopy including bronchoalveolar lavage (BAL) and transbronchial biopsy (TBB) offers a number of clear advantages, providing the most effective means to a rapid, accurate and comprehensive evaluation and diagnosis. In terms of ACR specifically, TBB can provide adequate tissue to facilitate histological evaluation. It continues-for now-to be considered the gold standard. Accepted practice is obtaining $\geq 5$ samples of well-expanded alveolated lung tissue (1), either from target lobes due to opacities on imaging or a lower lobe in the absence of radiological change $(63,64)$. Unilateral biopsies in a single intervention have long been considered prudent.

\section{Transbronchial cryobiopsy}

Given these concerns about specimen adequacy and diagnostic yield in conventional transbronchial biopsies, cryobiopsies have emerged as a realistic and potentially attractive alternative. Originally used for debulking endobronchial tumors, its application in transbronchial tissue sampling in suspected interstitial lung diseases was reported a decade ago (65). The procedure is usually performed in intubated patients and requires fluoroscopic guidance. The specialized probe tip is positioned with wall apposition and cooled to $-89^{\circ} \mathrm{C}$ for approximately 4 seconds. As the tissue samples are too large to pass through the working channel, the cryoprobe and flexible bronchoscope are removed $e n-b l o c$. Freezing is continued until the sample is removed from the patient. The first studies comparing cryobiospies to standard biopsies in LTx recipients appeared in 2013, suggesting a 3-5-fold increase in probe size when using cryobiopsy (66). Inevitably, larger probes results in greater trauma at the site of biopsy. Published reports among some early adopters frequent, severe complications particularly with regard to bleeding (67). Adaptions in techniques, particularly the prophylactic use of in situ bronchial blockers and specialized endotracheal tubes $(68,69)$ have led to significant improvements in safety data, with experienced centers now reporting equivalent safety profiles between techniques (70).

\section{Pathology/grading}

As stated above, central to the diagnosis of ACR and its grade is de novo lymphocyte perivascular infiltration within the allograft. Coordinated expert consensus was reached and subsequently adopted by the ISHLT in 1990 (71) and since then a number of revisions have taken place, the last of which was published in 2007 (1).

The classification employed allows independent reporting of the nature and semi-quantitative degree of mononuclear 
Table 1 Semi-quantitative summary of the distinguishing features, in terms of sites of inflammation and the respective cell types involved, used to classify acute cellular rejection according to the International Society For Heart \& Lung Transplantation (ISHLT) consensus document (1)

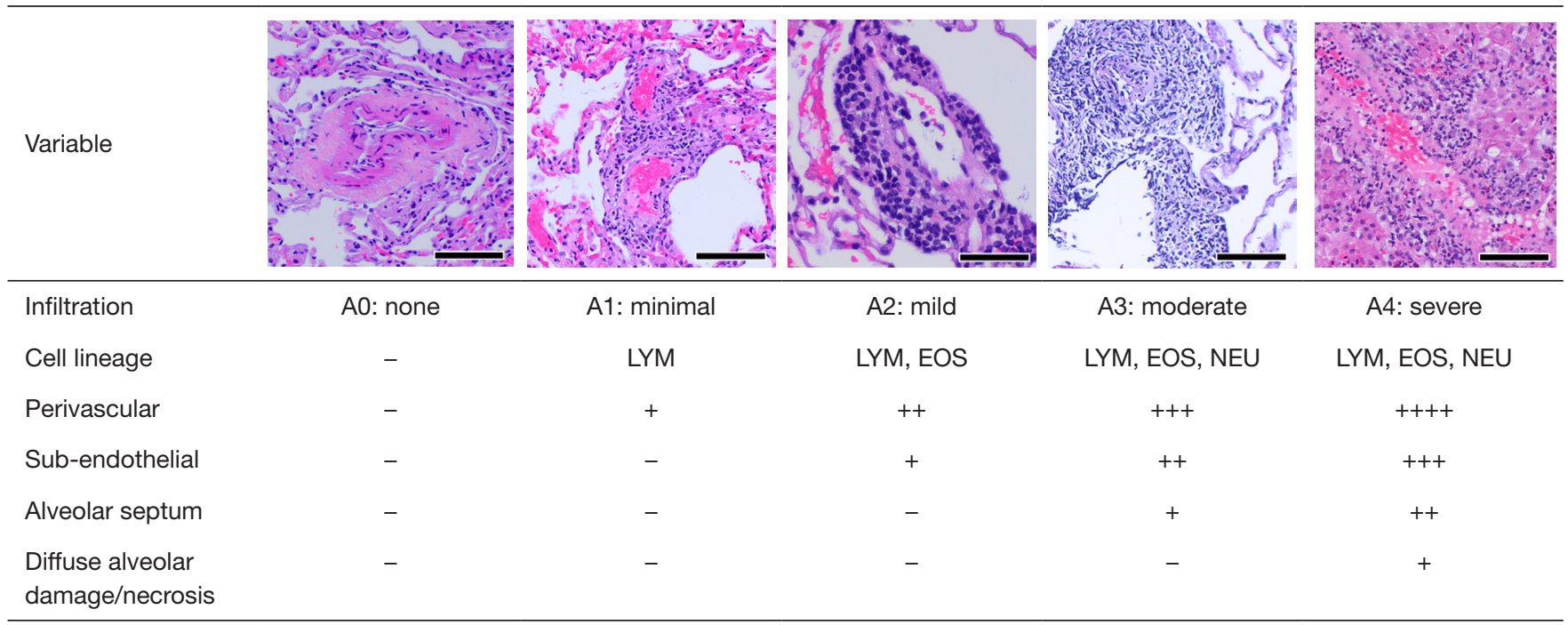

All images taken at 100x magnification and supplied courtesy of Institute for Pathology, Hannover Medical School, Hannover, Germany. LYM, lymphocytes; EOS, eosinophils; NEU, neutrophils.

Table 2 Semi-quantitative summary of the distinguishing features, in terms of sites of inflammation and the respective cell types involved, used to classify lymphocytic bronchitis observed in transbronchial biopsies according to the International Society For Heart \& Lung Transplantation (ISHLT) consensus document (1)

\begin{tabular}{|c|c|c|c|c|}
\hline Variable & 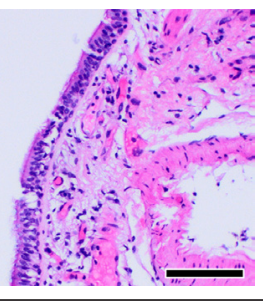 & 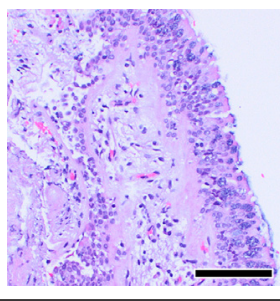 & 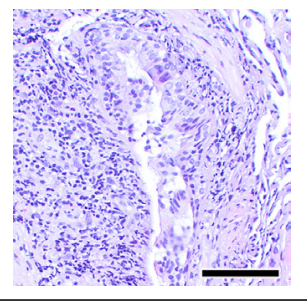 & (f) \\
\hline Infiltration & B0: none & B1R: low grade & B2R: high grade & BX: ungraded \\
\hline Cell lineage & & LYM, EOS(-) & LYM, EOS(++), NEU(-) & Sampling artifact, infection \\
\hline Sub-mucosal & - & + & ++ & \\
\hline Intra-epithelial & - & - & + & \\
\hline Necrosis/exudates & - & - & $-/+$ & \\
\hline
\end{tabular}

All images taken at 100x magnification and supplied courtesy of Institute for Pathology, Hannover Medical School, Hannover, Germany. B1R, Revised 2007 definition of B1; B2R, Revised 2007 definition of B2; LYM, lymphocytes; EOS, eosinoptablehils; NEU, neutrophils.

cell infiltration around blood vessels and adjacent alveolar parenchyma as well as smaller and larger airways. Ancillary information relating to coexisting, sub-acute processes such as obliterative bronchiolitis and premature allograft vascular sclerosis were routinely reported within the same biopsies. The grading used is summarized in Tables 1 and 2. In terms of interstitial changes, patchy perivascular cuffing- in particular venules-by lymphocytes are considered the first sign. Progressing lesions leading to involvement of arterioles as well as the adjacent sub-endothelium and the arrival of additional eosinophils herald a change from minimal to mild acute rejection. Further transition into a predominating endothelialitis with involvement of peribronchiolar alveolar septa and airspaces is diagnostic of 
moderate ACR. In this setting lymphocytes and eosinophils are joined by neutrophils. Beyond this, damage of the alveolar pneumocytes within a diffuse endothelialitis along with the accompanying changes above is considered severe ACR and can be associated with necrotic debris within the alveoli, hemorrhage and development of hyaline membranes.

A traditional accompaniment to assessing parenchymal perivascular status, similar inflammation of neighboring airways is included in reporting (Table 2). Due to poor reproducibility (72), consensus on quantifying lymphocytic bronchiolitis (LB) has been simplified over subsequent iterations of the classification and, when present, is currently considered as low or high-grade. Distinguishing between the two depends on progression of the mononuclear infiltration beyond the basement membrane, leading to epithelial damage and necrosis. Again cell populations shift towards other linages, with eosinophils becoming particularly prevalent as well as neutrophils. The direct link and indeed relevance of LB to ACR remains unclear. Limited data from a large single center cohort in which simultaneous large airway mucosal biopsies and transbronchial biopsies were performed during initial surveillance showed some concordance in LB and concomitant lymphocytic infiltration of larger airways (73) which had previously been associated with risk of developing chronic lung allograft dysfunction (CLAD) (74).

\section{Gold standard or shades of grey?}

With the exception of some simplification of B-grading mentioned above, the consensus recommendations have become well accepted in the LTx community and remain universally implemented in standard practice. They attempt to provide an objective end-point, but require bronchoscopy and the incumbent risks of performing trans-bronchial biopsies. However reproducibility and inter-observer agreement have generated some consternation.

Early single institution studies however revealed mixed inter-observer agreement, particularly with regard to quantification of more subtle forms of perivascular infiltration $(75,76)$, with inter-pathologist agreement revealing concordance rates at best of between $75-80 \%$. A-Grades have generally been shown to be more reliable than B-Grades (72). Such concerns led to a much larger analysis of over 1,500 biopsies from 845 patients in the Lung Allograft Rejection Gene Expression Observational Study (77). Here biopsies were again graded locally, before undergoing consensus regrading by an international panel of 2 from 3 nominated pathologists. Inter-observer agreement here was disappointingly low, with the highest concordance seen in $\mathrm{A} 0$ and $\geq \mathrm{A} 2$ groups where agreement was reached in half of the samples. Just over a fifth of A1 probes were confirmed and over one-third of center-diagnosed biopsies were deemed by the panel to be uninterpretable (77). In terms of treatment, considerable consequences with regard to under-treatment were noted. A subsequent, similar analysis again within a multi-center study, involving blinded review after center interpretations of almost 500 TBBs by a single pathologist (78). A-grade concordance was evident again in only 3 from 4 samples and became more discordant as time after LTx increased. Clearly, sample quality has often been cited as a confounding factor and cryobiopsies have been proposed as a viable alternative. Single center data comparing simultaneously obtained conventional TBB and cryobiopsy assessed independently, led to improvements in conclusive diagnosis from $78 \%$ to $97 \%$ (79). A followup study, in which cryobiopsy was implemented in the surveillance of asymptomatic patients, sample adequacy was reported at $98 \%$ when $\geq 4$ probes taken. ACR rates in year 1 were high than in TBB cohorts at $42 \%$, almost half of whom were asymptomatic. Of this sub-group two-thirds were $\geq \mathrm{A} 2$ (80).

\section{Surveillance}

Clearly the case for clinically-indicated bronchoscopy and TBB is strong. Less certainty remains on the merits of control bronchoscopy subsequent to complete clinical recovery and indeed surveillance bronchoscopy and biopsy at scheduled time points after LTx, independent of clinical course.

Well established arguments both for and against surveillance have been presented previously $(63,81-84)$. While the relatively high detection rates of clinically asymptomatic ACR is the principal justification for surveillance. The relevance of these findings to long-term outcomes has been questioned $(85,86)$ and unnecessary exposure of apparently stable patients to procedural risk are often cited as the main counterarguments. Previous studies have revealed great inter-institutional variability in follow-up practices, with approximately $50 \%$ performing surveillance biopsies in the first year at the time (86). Compounding clinical aspects, strategies will be strongly influenced by center volume, institutional infrastructure, patient dispersion and reimbursement. 
In appraising the clinical arguments related to surveillance bronchoscopy, thorough examination of the evidence base is essential in quantifying risk. Procedural risks vary according to local practice, but essentially consist of transient hypoxia, bleeding, pneumothorax, postinterventional fever and sepsis $(51,81,86,87)$. Transient hypoxia is consistently reported as the most common complication of bronchoscopy in LTx recipients, affecting as many as 1 in 20 to 1 in 10 patients $(63,81,88)$. The respective centers all employed conscious sedation, based upon individual titration of various intravenous sedatives without airway adjuncts. In most cases oxygen desaturation resulted from occlusion of the upper airways and was instantly remedied by insertion of airway adjuncts such as naso-pharyngeal tubes. Even in the absence of complications, such patients require careful post-sedation monitoring, necessitating dedicated staff, a recovery bay and in many cases an overnight stay due to the logistics involved. Such system demands have led some centers to attempt flexible bronchoscopy including TBB on an outpatient basis without routine use of sedation (89). Reporting on over 3,000 procedures, almost $80 \%$ were performed without complication, the most common complication being cough $(5.3 \%)$, with oxygen desaturation being recorded in $2 \%$. Procedure duration was median 15 minutes, and patients were discharged home within 2 hours of the procedure. In total only 4 procedures led to hospitalization.

Bleeding rates post biopsy of varying incidence have been reported, but in the three studies assessing safety in LTx recipients directly rates of between $4-13 \%$ were reported $(51,81,89)$. It should be noted, that these rates reflected any bleeding, with no reports of bleeding refractory to local topical treatment measures. In experienced hands, cryobiopsies are reported to demonstrate similar bleeding complications as standard TBB (70).

Pneumothorax rates of between $0.1-4 \%$ were reported in the same studies $(51,70,81,89)$, with only a small proportion requiring chest tube insertion. Again in direct comparison, no differences between TBB and cryobiopsy in experienced centers were observed (70). Interestingly, centers performing TBB in non-sedated patients did so without using fluoroscopic guidance, relying instead on patient feedback. In this report the pneumothorax rate was $0.4 \%$ and with a biopsy yield for ISHLT A-grading of 96.2\% (89).

Risk of fever, pneumonia and sepsis as a result of bronchoscopy within the LTx populations are often included in the argument against surveillance, but very little published data exists. In non-LTx populations, post-procedural fever has been reported in up to $16 \%$ of immunocompetent adults $(90,91)$, with transient bacteremia (92) and cytokine release from alveolar macrophages (93) being postulated causes. Novel use of RNA sequencing in peripheral blood has recently been shown to augment investigation in affected patients, to identify bacteria in culture negative or even colonized patients (90).

\section{Treatment}

The other main contention about ACR, which also feeds into the surveillance debate, is the necessity of treatment in asymptomatic patients and indeed its long-term implications. According to ISHLT data, ACR accounts for less than $2 \%$ of all deaths at any given time point beyond the first month (2). Beyond acute symptoms, its principal implication is the body of evidence associating ACR with increased risk of CLAD (94-96). For symptomatic ACR grades $\geq$ A2 in the absence of contributory causes, widespread consensus for pulsed intravenous steroids for around 3 consecutive days exists. Complete restoration of graft function within a matter of days is anticipated. For milder grades, both with or without symptoms in presence of allograft dysfunction no data or consensus exists, with some centers advocating oral steroids $(0.5-1.0 \mathrm{mg} / \mathrm{kg}$ Prednisolone) and others employing watchful waiting with re-biopsy at 4-6 weeks. Conflicting evidence linking mild ACR (A1) to CLAD has been published previously, with some centers considering even a single episode to confer risk and warrant treatment $(95,97)$. These findings however, have never been unequivocal, with others finding no meaningful association $(73,98)$. A recent single center study, examining watchful waiting for spirometrically stable A1 in 173 patients observed no differences in either graft or CLAD-free survival, compared to matched controls who had never experienced biopsy-proven ACR (99). Most centers undertaking surveillance biopsies usually perform control biopsies in treatment requiring ACR, again at 4-6 weeks with persisting ACR rates of up to $26 \%$ being cited as justification (100).

A lack of both evidence and consensus exists in the management of steroid-refractory ACR. Most centers appear to repeat steroid pulses, potentially at higher doses. Changes in maintenance immunosuppression, particularly switching to Tacrolimus among those receiving Cyclosporine (101) and the introduction of mTOR inhibitors such as Sirolimus or Everolimus have been reported. Although data for the latter in other solid organ transplantation exists, LTx-specific 
benefits have not been shown (102). Beyond this, single center studies have reported success with Alemtuzumab (103), Cyclophosphamide (104) and extracorporeal Photopheresis (105). In such cases however, the most important issue is reappraisal of the individual clinical data and reconsideration of possible coexisting issues including antibody-mediated rejection (AMR).

\section{Potential alternative biomarkers}

Given the universal use of BAL, it has been extensively studied as a surrogate for TBB. Flow cytometry analysis of BAL samples has suggested correlating patterns of monocyte, NK cells and specific lymphocyte subtypes with ACR (106). Analyzing gene expression in cell pellets derived from BAL as a surrogate for TBB has been suggested, and preliminary gene expression profiles for A-grade ACR postulated (107). Quantitative analysis of BAL however has its own inherent limitations (108) and standardization of protocols appears necessary.

Serum markers offer the greatest attraction and a myriad of potential markers have been suggested but reproducibility has proved elusive. Donor-derived cell-free DNA has shown the most promise in detecting allograft injury (109). Scientific advances to facilitate discrimination between different causes of cell death have been reported and technological advances to facilitate rapid detection are the current focus of development (110).

\section{Conclusions}

Although ACR occurs frequently in the early posttransplant course, timely identification and treatment of symptomatic cases generally respond favorably to high-dose steroids. Extrinsic factors as well as intrinsic variations in all aspects of alloimmune compatibility appear to influence the likelihood of developing ACR. ACR-related mortality is very low, with the main concern being its association with subsequent development of CLAD. Clinical manifestations are variable and non-specific, necessitating comprehensive diagnostic evaluation. At present, diagnosis requires allograft biopsy for confirmation. A well-defined classification system exists, but inter-observer variability limits its use in multicenter studies. The relevance of asymptomatic and mild grade ACR is contentious, and the risk of allograft injury must offset the anticipated morbidity associated with the proposed treatment intervention. Intertwined with this is the additional contention regarding the utility of biopsy surveillance in LTx, despite the increasingly acceptable risk profile of the procedure as experiences improves. Minimal and non-invasive alternative biomarkers have been proposed, but so far none have surpassed tissue biopsies. This however remains an area of ongoing research.

\section{Acknowledgments}

Funding: None.

\section{Footnote}

Provenance and Peer Review: This article was commissioned by the Guest Editors (Masaaki Sato and Dong Tian) for the series "Strategies to Achieve Long-Term Success of Lung Transplantation" published in Annals of Translational Medicine. The article was sent for external peer review organized by the Guest Editors and the editorial office.

Conflicts of Interest: The series "Strategies to Achieve LongTerm Success of Lung Transplantation" was commissioned by the editorial office without any funding or sponsorship. The authors have no conflicts of interest to declare.

Ethical Statement: The authors are accountable for all aspects of the work in ensuring that questions related to the accuracy or integrity of any part of the work are appropriately investigated and resolved.

Open Access Statement: This is an Open Access article distributed in accordance with the Creative Commons Attribution-NonCommercial-NoDerivs 4.0 International License (CC BY-NC-ND 4.0), which permits the noncommercial replication and distribution of the article with the strict proviso that no changes or edits are made and the original work is properly cited (including links to both the formal publication through the relevant DOI and the license). See: https://creativecommons.org/licenses/by-nc-nd/4.0/.

\section{References}

1. Stewart S, Fishbein MC, Snell GI, et al. Revision of the 1996 working formulation for the standardization of nomenclature in the diagnosis of lung rejection. J Heart Lung Transplant 2007;26:1229-42.

2. Chambers DC, Cherikh WS, Harhay MO, et al. The International Thoracic Organ Transplant Registry of the International Society for Heart and Lung 
Transplantation: Thirty-sixth adult lung and heartlung transplantation Report-2019; Focus theme: Donor and recipient size match. J Heart Lung Transplant 2019;38:1042-55.

3. Glanville AR, Aboyoun C, Klepetko W, et al. Threeyear results of an investigator-driven multicenter, international, randomized open-label de novo trial to prevent BOS after lung transplantation. J Heart Lung Transplant 2015;34:16-25.

4. Hachem RR, Yusen RD, Chakinala MM, et al. A randomized controlled trial of tacrolimus versus cyclosporine after lung transplantation. J Heart Lung Transplant 2007;26:1012-8.

5. Lafferty KJ, Bootes A, Dart G, et al. Effect of organ culture on the survival of thyroid allografts in mice. Transplantation 1976;22:138-49.

6. Ingulli E. Mechanism of cellular rejection in transplantation. Pediatr Nephrol 2010;25:61-74.

7. Koutsokera A, Levy L, Pal P, et al. Acute Cellular Rejection: Is It Still Relevant? Semin Respir Crit Care Med 2018;39:181-98.

8. Salcedo-Herrera S, Pinto Ramirez JL, Garcia-Lopez A, et al. Acute Rejection in Kidney Transplantation and Early Beginning of Tacrolimus. Transplant Proc 2019;51:1758-62.

9. Shaked A, Ghobrial RM, Merion RM, et al. Incidence and severity of acute cellular rejection in recipients undergoing adult living donor or deceased donor liver transplantation. Am J Transplant 2009;9:301-8.

10. Snell GI, Levvey BJ, Paraskeva M, et al. The influence of clinical donor factors on acute rejection among lung and kidney recipients from the same multi-organ donor. Ann Transplant 2013;18:358-67.

11. Calabrese F, Lunardi F, Nannini N, et al. Higher Risk of Acute Cellular Rejection in Lung Transplant Recipients with Cystic Fibrosis. Ann Transplant 2015;20:769-76.

12. Penninga L, Moller CH, Penninga EI, et al. Antibody induction therapy for lung transplant recipients. Cochrane Database Syst Rev 2013;(11):CD008927.

13. Li KHC, Ho JCS, Recaldin B, et al. Acute Cellular Rejection and Infection Rates in Alemtuzumab vs Traditional Induction Therapy Agents for Lung and Heart Transplantation: A Systematic Review and Meta-analysis. Transplant Proc 2018;50:3723-31.

14. Hu L, Lingler JH, Sereika SM, et al. Nonadherence to the medical regimen after lung transplantation: A systematic review. Heart Lung 2017;46:178-86.

15. Kugler C, Fuehner T, Dierich M, et al. Effect of adherence to home spirometry on bronchiolitis obliterans and graft survival after lung transplantation. Transplantation 2009;88:129-34.

16. Geramita EM, DeVito Dabbs AJ, DiMartini AF, et al. Impact of a Mobile Health Intervention on Long-Term Nonadherence After Lung Transplantation: Follow-up After a Randomized Controlled Trial. Transplantation 2020;104:640-51.

17. Quantz MA, Bennett LE, Meyer DM, et al. Does human leukocyte antigen matching influence the outcome of lung transplantation? An analysis of 3,549 lung transplantations. J Heart Lung Transplant 2000;19:473-9.

18. Reinsmoen NL, Mirocha J, Ensor CR, et al. A 3-Center Study Reveals New Insights Into the Impact of NonHLA Antibodies on Lung Transplantation Outcome. Transplantation 2017;101:1215-21.

19. Colobran R, Casamitjana N, Roman A, et al. Copy number variation in the CCL4L gene is associated with susceptibility to acute rejection in lung transplantation. Genes Immun 2009;10:254-9.

20. Barton GM, Medzhitov R. Control of adaptive immune responses by Toll-like receptors. Curr Opin Immunol 2002;14:380-3.

21. Arbour NC, Lorenz E, Schutte BC, et al. TLR4 mutations are associated with endotoxin hyporesponsiveness in humans. Nat Genet 2000;25:187-91.

22. Girnita DM, Webber SA, Zeevi A. Clinical impact of cytokine and growth factor genetic polymorphisms in thoracic organ transplantation. Clin Lab Med 2008;28:423-40, vi.

23. Palmer SM, Burch LH, Davis RD, et al. The role of innate immunity in acute allograft rejection after lung transplantation. Am J Respir Crit Care Med 2003;168:628-32.

24. Kardol-Hoefnagel T, Budding K, van de Graaf EA, et al. A Single Nucleotide C3 Polymorphism Associates With Clinical Outcome After Lung Transplantation. Front Immunol 2019;10:2245.

25. Paul P, Pedini P, Lyonnet L, et al. FCGR3A and FCGR2A Genotypes Differentially Impact Allograft Rejection and Patients' Survival After Lung Transplant. Front Immunol 2019;10:1208.

26. Tague LK, Byers DE, Hachem R, et al. Impact of SLCO1B3 polymorphisms on clinical outcomes in lung allograft recipients receiving mycophenolic acid. Pharmacogenomics J 2020;20:69-79.

27. Roux A, Mourin G, Fastenackels S, et al. CMV driven CD8(+) T-cell activation is associated with 
acute rejection in lung transplantation. Clin Immunol 2013;148:16-26.

28. Streblow DN, Orloff SL, Nelson JA. Acceleration of allograft failure by cytomegalovirus. Curr Opin Immunol 2007;19:577-82.

29. McLaughlin K, Wu C, Fick G, et al. Cytomegalovirus seromismatching increases the risk of acute renal allograft rejection. Transplantation 2002;74:813-6.

30. Chang A, Musk M, Lavender M, et al. Cytomegalovirus viremia in lung transplantation during and after prophylaxis. Transpl Infect Dis 2019;21:e13069.

31. Heliövaara E, Husain S, Martinu T, et al. Drug-resistant cytomegalovirus infection after lung transplantation: Incidence, characteristics, and clinical outcomes. J Heart Lung Transplant 2019;38:1268-74.

32. Bridevaux PO, Aubert JD, Soccal PM, et al. Incidence and outcomes of respiratory viral infections in lung transplant recipients: a prospective study. Thorax 2014;69:32-8.

33. Magnusson J, Westin J, Andersson LM, et al. Viral Respiratory Tract Infection During the First Postoperative Year Is a Risk Factor for Chronic Rejection After Lung Transplantation. Transplant Direct 2018;4:e370.

34. Soccal PM, Aubert JD, Bridevaux PO, et al. Upper and lower respiratory tract viral infections and acute graft rejection in lung transplant recipients. Clin Infect Dis 2010;51:163-70.

35. Vu DL, Bridevaux PO, Aubert JD, et al. Respiratory viruses in lung transplant recipients: a critical review and pooled analysis of clinical studies. Am J Transplant 2011;11:1071-8.

36. Peghin M, Los-Arcos I, Hirsch HH, et al. Communityacquired Respiratory Viruses Are a Risk Factor for Chronic Lung Allograft Dysfunction. Clin Infect Dis 2019;69:1192-7.

37. Mohanakumar T, Sharma M, Bansal S, et al. A novel mechanism for immune regulation after human lung transplantation. J Thorac Cardiovasc Surg 2019;157:2096-106.

38. Ravichandran R, Bansal S, Rahman M, et al. The role of donor-derived exosomes in lung allograft rejection. Hum Immunol 2019;80:588-94.

39. Tague LK, Scozzi D, Wallendorf M, et al. Lung transplant outcomes are influenced by severity of neutropenia and granulocyte colony-stimulating factor treatment. Am J Transplant 2020;20:250-61.

40. Casciello N, Hulbert A, Snyder L, et al. Incidence of acute cellular rejection following granulocyte colonystimulating factor administration in lung transplantation: A retrospective case-cohort analysis. Clin Transplant 2017. doi: 10.1111/ctr.12965.

41. Verleden SE, Gottlieb J, Dubbeldam A, et al. "WhiteOut" After Lung Transplantation: A Multicenter Cohort Description of Late Acute Graft Failure. Am J Transplant 2017;17:1905-11.

42. De Vito Dabbs A, Hoffman LA, Iacono AT, et al. Are symptom reports useful for differentiating between acute rejection and pulmonary infection after lung transplantation? Heart Lung 2004;33:372-80.

43. Speck NE, Schuurmans MM, Murer C, et al. Diagnostic value of plasma and bronchoalveolar lavage samples in acute lung allograft rejection: differential cytology. Respir Res 2016;17:74.

44. Tikkanen J, Lemstrom K, Halme M, et al. Cytological monitoring of peripheral blood, bronchoalveolar lavage fluid, and transbronchial biopsy specimens during acute rejection and cytomegalovirus infection in lung and heart--lung allograft recipients. Clin Transplant 2001;15:77-88.

45. Tikkanen J, Lemstrom K, Halme M, et al. Detailed analysis of cell profiles in peripheral blood, bronchoalveolar lavage fluid, and transbronchial biopsy specimens during acute rejection and CMV infection in lung and heart-lung allograft recipients. Transplant Proc 1999;31:163-4.

46. Trull A, Steel L, Cornelissen J, et al. Association between blood eosinophil counts and acute cardiac and pulmonary allograft rejection. J Heart Lung Transplant 1998;17:517-24.

47. Otulana BA, Higenbottam T, Scott J, et al. Lung function associated with histologically diagnosed acute lung rejection and pulmonary infection in heart-lung transplant patients. Am Rev Respir Dis 1990;142:329-32.

48. Otulana BA, Higenbottam T, Ferrari L, et al. The use of home spirometry in detecting acute lung rejection and infection following heart-lung transplantation. Chest 1990;97:353-7.

49. Sengpiel J, Fuehner T, Kugler C, et al. Use of telehealth technology for home spirometry after lung transplantation: a randomized controlled trial. Prog Transplant 2010;20:310-7.

50. Becker FS, Martinez FJ, Brunsting LA, et al. Limitations of spirometry in detecting rejection after single-lung transplantation. Am J Respir Crit Care Med 1994;150:159-66.

51. Hopkins PM, Aboyoun CL, Chhajed PN, et al. 
Prospective analysis of 1,235 transbronchial lung biopsies in lung transplant recipients. J Heart Lung Transplant 2002;21:1062-7.

52. Gotway MB, Dawn SK, Sellami D, et al. Acute rejection following lung transplantation: limitations in accuracy of thin-section CT for diagnosis. Radiology 2001;221:207-12.

53. Park CH, Paik HC, Haam SJ, et al. HRCT features of acute rejection in patients with bilateral lung transplantation: the usefulness of lesion distribution. Transplant Proc 2014;46:1511-6.

54. Di Piazza A, Mamone G, Caruso S, et al. Acute rejection after lung transplantation: association between histopathological and CT findings. Radiol Med 2019;124:1000-5.

55. Martinu T, Chen DF, Palmer SM. Acute rejection and humoral sensitization in lung transplant recipients. Proc Am Thorac Soc 2009;6:54-65.

56. Chuck NC, Boss A, Wurnig MC, et al. Ultra-short echotime magnetic resonance imaging distinguishes ischemia/ reperfusion injury from acute rejection in a mouse lung transplantation model. Transpl Int 2016;29:108-18.

57. Siddiqui S, Habertheuer A, Xin Y, et al. Detection of lung transplant rejection in a rat model using hyperpolarized [1(13) C] pyruvate-based metabolic imaging. NMR Biomed 2019;32:e4107.

58. Telenga ED, van der Bij W, de Vries EFJ, et al. (99m)TcHYNIC-IL-2 scintigraphy to detect acute rejection in lung transplantation patients: a proof-of-concept study. EJNMMI Res 2019;9:41.

59. Jones HA, Donovan T, Goddard MJ, et al. Use of 18FDGpet to discriminate between infection and rejection in lung transplant recipients. Transplantation 2004;77:1462-4.

60. Fuehner T, Dierich M, Duesberg C, et al. Endoscopic indicators for obstructive airway complications after lung transplantation. Transplantation 2010;90:1210-4.

61. Kroegel C, Hekmat K, Moser A, et al. Airway complications following lung transplantation - clinic, diagnosis, and interventional management. Pneumologie 2011;65:293-307.

62. Murthy SC, Blackstone EH, Gildea TR, et al. Impact of anastomotic airway complications after lung transplantation. Ann Thorac Surg 2007;84:401-9, 9 e1-4.

63. Benzimra M, Calligaro GL, Glanville AR. Acute rejection. J Thorac Dis 2017;9:5440-57.

64. Hasegawa T, Iacono AT, Yousem SA. The anatomic distribution of acute cellular rejection in the allograft lung. Ann Thorac Surg 2000;69:1529-31.

65. Babiak A, Hetzel J, Krishna G, et al. Transbronchial cryobiopsy: a new tool for lung biopsies. Respiration 2009; 78:203-8.

66. Fruchter O, Fridel L, Rosengarten D, et al. Transbronchial cryo-biopsy in lung transplantation patients: first report. Respirology 2013;18:669-73.

67. DiBardino DM, Haas AR, Lanfranco AR, et al. High Complication Rate after Introduction of Transbronchial Cryobiopsy into Clinical Practice at an Academic Medical Center. Ann Am Thorac Soc 2017;14:851-7.

68. Sastre JA, Cordovilla R, Jimenez MF, et al. Management of a transbronchial cryobiopsy using the i-gel(R) airway and the Arndt endobronchial blocker. Can J Anaesth 2014;61:886-8.

69. Ravaglia C, Bonifazi M, Wells AU, et al. Safety and Diagnostic Yield of Transbronchial Lung Cryobiopsy in Diffuse Parenchymal Lung Diseases: A Comparative Study versus Video-Assisted Thoracoscopic Lung Biopsy and a Systematic Review of the Literature. Respiration 2016;91:215-27.

70. Gershman E, Ridman E, Fridel L, et al. Efficacy and safety of trans-bronchial cryo in comparison with forceps biopsy in lung allograft recipients: Analysis of 402 procedures. Clin Transplant 2018;32:e13221.

71. Berry GJ, Brunt EM, Chamberlain D, et al. A working formulation for the standardization of nomenclature in the diagnosis of heart and lung rejection: Lung Rejection Study Group. The International Society for Heart Transplantation. J Heart Transplant 1990;9:593-601.

72. Chakinala MM, Ritter J, Gage BF, et al. Reliability for grading acute rejection and airway inflammation after lung transplantation. J Heart Lung Transplant 2005;24:652-7.

73. Greenland JR, Jones KD, Hays SR, et al. Association of large-airway lymphocytic bronchitis with bronchiolitis obliterans syndrome. Am J Respir Crit Care Med 2013;187:417-23.

74. Glanville AR, Aboyoun CL, Havryk A, et al. Severity of lymphocytic bronchiolitis predicts long-term outcome after lung transplantation. Am J Respir Crit Care Med 2008;177:1033-40.

75. Stephenson A, Flint J, English J, et al. Interpretation of transbronchial lung biopsies from lung transplant recipients: inter- and intraobserver agreement. Can Respir J 2005;12:75-7.

76. Colombat M, Groussard O, Lautrette A, et al. Analysis of the different histologic lesions observed in transbronchial biopsy for the diagnosis of acute rejection. Clinicopathologic correlations during the first 6 months after lung transplantation. Hum Pathol 2005;36:387-94. 
77. Arcasoy SM, Berry G, Marboe CC, et al. Pathologic interpretation of transbronchial biopsy for acute rejection of lung allograft is highly variable. Am J Transplant 2011;11:320-8.

78. Bhorade SM, Husain AN, Liao C, et al. Interobserver variability in grading transbronchial lung biopsy specimens after lung transplantation. Chest 2013;143:1717-24.

79. Montero MA, de Gracia J, Culebras Amigo M, et al. The role of transbronchial cryobiopsy in lung transplantation. Histopathology 2018;73:593-600.

80. Loor K, Culebras M, Sansano I, et al. Optimization of Transbronchial Cryobiopsy in Lung Transplant Recipients. Ann Thorac Surg 2019;108:1052-8.

81. McWilliams TJ, Williams TJ, Whitford HM, et al. Surveillance bronchoscopy in lung transplant recipients: risk versus benefit. J Heart Lung Transplant 2008;27:1203-9.

82. Glanville AR. The role of surveillance bronchoscopy post-lung transplantation. Semin Respir Crit Care Med 2013;34:414-20.

83. Glanville AR. Bronchoscopic monitoring after lung transplantation. Semin Respir Crit Care Med 2010;31:208-21.

84. Benzimra M. Surveillance Bronchoscopy: Is It Still Relevant? Semin Respir Crit Care Med 2018;39:219-26.

85. Valentine VG, Taylor DE, Dhillon GS, et al. Success of lung transplantation without surveillance bronchoscopy. J Heart Lung Transplant 2002;21:319-26.

86. Valentine VG, Gupta MR, Weill D, et al. Singleinstitution study evaluating the utility of surveillance bronchoscopy after lung transplantation. J Heart Lung Transplant 2009;28:14-20.

87. Chakinala MM, Ritter J, Gage BF, et al. Yield of surveillance bronchoscopy for acute rejection and lymphocytic bronchitis/bronchiolitis after lung transplantation. J Heart Lung Transplant 2004;23:1396-404.

88. Dransfield MT, Garver RI, Weill D. Standardized guidelines for surveillance bronchoscopy reduce complications in lung transplant recipients. J Heart Lung Transplant 2004;23:110-4.

89. Rademacher J, Suhling H, Greer M, et al. Safety and efficacy of outpatient bronchoscopy in lung transplant recipients - a single centre analysis of 3,197 procedures. Transplant Res 2014;3:11.

90. Ko ER, Philipson CW, Burke TW, et al. Direct-fromblood RNA sequencing identifies the cause of postbronchoscopy fever. BMC Infect Dis 2019;19:905.

91. Um SW, Choi CM, Lee CT, et al. Prospective analysis of clinical characteristics and risk factors of postbronchoscopy fever. Chest 2004;125:945-52.

92. Alexander WJ, Baker GL, Hunker FD. Bacteremia and meningitis following fiberoptic bronchoscopy. Arch Intern Med 1979;139:580-3.

93. Krause A, Hohberg B, Heine F, et al. Cytokines derived from alveolar macrophages induce fever after bronchoscopy and bronchoalveolar lavage. Am J Respir Crit Care Med 1997;155:1793-7.

94. Burton CM, Iversen M, Carlsen J, et al. Acute cellular rejection is a risk factor for bronchiolitis obliterans syndrome independent of post-transplant baseline FEV1. J Heart Lung Transplant 2009;28:888-93.

95. Khalifah AP, Hachem RR, Chakinala MM, et al. Minimal acute rejection after lung transplantation: a risk for bronchiolitis obliterans syndrome. Am J Transplant 2005;5:2022-30.

96. Hopkins PM, Aboyoun CL, Chhajed PN, et al. Association of minimal rejection in lung transplant recipients with obliterative bronchiolitis. Am J Respir Crit Care Med 2004;170:1022-6.

97. Hachem RR, Khalifah AP, Chakinala MM, et al. The significance of a single episode of minimal acute rejection after lung transplantation. Transplantation 2005;80:1406-13.

98. Schulman LL, Weinberg AD, McGregor C, et al. Mismatches at the HLA-DR and HLA-B loci are risk factors for acute rejection after lung transplantation. Am J Respir Crit Care Med 1998;157:1833-7.

99. Levy L, Huszti E, Tikkanen J, et al. The impact of first untreated subclinical minimal acute rejection on risk for chronic lung allograft dysfunction or death after lung transplantation. Am J Transplant 2020;20:241-9.

100. Aboyoun CL, Tamm M, Chhajed PN, et al. Diagnostic value of follow-up transbronchial lung biopsy after lung rejection. Am J Respir Crit Care Med 2001;164:460-3.

101. Sarahrudi K, Estenne M, Corris P, et al. International experience with conversion from cyclosporine to tacrolimus for acute and chronic lung allograft rejection. J Thorac Cardiovasc Surg 2004;127:1126-32.

102. Bhorade S, Ahya VN, Baz MA, et al. Comparison of sirolimus with azathioprine in a tacrolimus-based immunosuppressive regimen in lung transplantation. Am J Respir Crit Care Med 2011;183:379-87.

103. Reams BD, Musselwhite LW, Zaas DW, et al. Alemtuzumab in the treatment of refractory acute rejection and bronchiolitis obliterans syndrome after human lung transplantation. Am J Transplant 2007;7:2802-8. 
104. Naik C, Moore C, Pipeling M, et al. Cyclophosphamide for Refractory Acute Cellular Rejection After Lung Transplantation. Transplant Direct 2018;4:e350.

105.Benden C, Speich R, Hofbauer GF, et al. Extracorporeal photopheresis after lung transplantation: a 10-year singlecenter experience. Transplantation 2008;86:1625-7.

106. Greenland JR, Jewell NP, Gottschall M, et al. Bronchoalveolar lavage cell immunophenotyping facilitates diagnosis of lung allograft rejection. Am J Transplant 2014;14:831-40.

107. Weigt SS, Wang X, Palchevskiy V, et al. Usefulness of gene expression profiling of bronchoalveolar lavage cells in acute lung allograft rejection. J Heart Lung Transplant
2019;38:845-55.

108. Bollmann BA, Seeliger B, Drick N, et al. Cellular analysis in bronchoalveolar lavage: inherent limitations of current standard procedure. Eur Respir J 2017;49.

109. De Vlaminck I, Martin L, Kertesz M, et al. Noninvasive monitoring of infection and rejection after lung transplantation. Proc Natl Acad Sci U S A 2015;112:13336-41.

110.Zou J, Duffy B, Slade M, et al. Rapid detection of donor cell free DNA in lung transplant recipients with rejections using donor-recipient HLA mismatch. Hum Immunol 2017;78:342-9.
Cite this article as: Greer M, Werlein C, Jonigk D. Surveillance for acute cellular rejection after lung transplantation. Ann Transl Med 2020;8(6):410. doi: 10.21037/ atm.2020.02.127 